\title{
Ácidos graxos no desempenho e nas respostas imunológicas de bovinos Nelore confinados
}

\author{
Robson Sfaciotti Barducci(1), Luís Marcelo Nave Sarti(1), Danilo Domingues Millen(2), Thaila Cristina Putarov(1), \\ Felipe Azevedo Ribeiro( ${ }^{(1)}$, Maria Caroline da Silva Franzói(1), Carolina Floret da Costa ${ }^{(1)}$, \\ Cyntia Ludovico Martins ${ }^{(1)}$ e Mário De Beni Arrigoni(1)
}

\begin{abstract}
(1)Universidade Estadual Paulista, Departamento de Melhoramento e Nutrição Animal, Campus de Botucatu, Distrito Rubião Júnior, s/no, CEP 18618-000 Botucatu, SP, Brasil. E-mail: robsonbarducci@yahoo.com.br, Im.sarti@hotmail.com, thaputarov@yahoo.com.br, felipegea@hotmail.com, caroline_franzoi@yahoo.com.br, carolfloret@hotmail.com, cludovico@fmvz.unesp.br, arrigoni@fmvz.unesp.br (2)Universidade Estadual Paulista, Faculdade de Zootecnia, Campus de Dracena, Rodovia Comandante João Ribeiro de Barros, Km 651, CEP 17900-000 Dracena, SP, Brasil. E-mail: danilomillen@dracena.unesp.br
\end{abstract}

Resumo - O objetivo deste trabalho foi avaliar os efeitos da adição de fontes de lipídeos naturais e protegidos no desempenho e nas respostas imunológicas de bovinos Nelore em confinamento. Utilizou-se o delineamento experimental inteiramente casualizado com medidas repetidas no tempo e três tratamentos, que foram: sem fonte adicional de lipídeo (CONTR); com fonte de lipídeo natural (torta de algodão) (GDESP); e com fonte de lipídeo protegido e rico em ácidos graxos poli-insaturados (GPROT). O estudo foi dividido em duas fases: précondicionamento e confinamento de 120 bovinos Nelore inteiros (24 baias, 5 animais por baia e 8 repetições por tratamento). O tratamento GDESP proporcionou maior ganho de peso na fase de pré-condicionamento, e, durante o período de confinamento, não houve diferenças entre os tratamentos quanto ao desempenho, às características de carcaça e ao acometimento de enfermidades. As concentrações plasmáticas de TNF $\alpha$, IL- $1_{\beta}$, haptoglobina e ceruloplasmina foram superiores no tratamento CONTR. A adição de lipídeos à dieta, independentemente da fonte, promove melhora no desempenho e nos parâmetros imunológicos de bovinos Nelore confinados.

Termos para indexação: gordura protegida, pré-condicionamento, proteínas de fase aguda, Pufa, suplementação lipídica.

\section{Fatty acids on the performance and immune responses of Nellore feedlot cattle}

Abstract - The objective of this work was to evaluate the addition of natural and protected lipid sources on the performance and immune responses of Nelore cattle feedlot. A completely randomized design was carried out with repeated measures in time and three treatments, which were: without additional source of lipid (CONTR); with natural lipid source (cottonseed cake) (GDESP); and with rumen protected lipid source, rich in polyunsaturated fatty acids (GPROT). The study was divided into two phases: preconditioning and feedlot of 120 noncastrated Nelore cattle ( 24 pens, 5 animals per pen, and 8 pens per treatment). The GDESP treatment provided greater weight gain in the preconditioning phase, and, during the feedlot period, there were no differences among treatments for performance, carcass traits, and disease incidence. The highest plasma concentrations of TNF $\alpha, \mathrm{IL}_{1} 1_{\beta}$, haptoglobin, and ceruloplasmin occurred in the CONTR treatment. Lipid supplementation to the diet, regardless of the source, improves the performance and enhances the immunological system of confined Nellore cattle.

Index terms: rumen protected fat, preconditioning, acute phase proteins, Pufa, lipid suplementation.

\section{Introdução}

Nos últimos anos, a pecuária nacional intensificou seu sistema de produção, por meio do aumento de confinamentos. Entretanto, alguns processos que o antecedem, como o transporte às instalações, necessitam de atenção especial, pois são fatores estressores potenciais que afetam a saúde e o desempenho dos animais, como alterações fisiológicas, nutricionais e imunológicas (Loerch \& Fluharty, 1999).

Normalmente, no Brasil, o gado é transportado por longas distâncias até a chegada ao confinamento, o que gera condições de estresse e predisposição à queda do sistema imunológico (Cooke et al., 2011). Nesse contexto, o gado fica fragilizado e surgem enfermidades, como as broncopneumonias (Radostits et al., 2007). 
O fornecimento de ácidos graxos protegidos da degradação ruminal, ricos em ácidos poli-insaturados (AGPI), no período anterior ao transporte e no confinamento, ameniza os efeitos do estresse sobre o desempenho e favorece as condições do animal, principalmente pela modulação do sistema imune (Cooke et al., 2011). O manejo da dieta, antes do embarque, pode tornar-se prática importante para diminuir a morbidade e mortalidade de bovinos transportados, e a suplementação de AGPI poderá melhorar a resposta de proteínas de fase aguda, estimuladas pelo transporte e pela entrada no confinamento e, portanto, beneficiar o desempenho animal.

Vários estudos evidenciam o efeito positivo sobre as características de desempenho e respostas imunológicas dos animais, quando há inclusão de lipídeos em dietas para bovinos, durante o transporte e confinamento (Arthington et al., 2005; Arthington et al., 2008; Farran et al., 2008; Araujo et al., 2010; Cooke et al., 2010). No entanto, a suplementação de AGPI, como sabões de cálcio, pode prejudicar a ingestão de matéria seca e afetar outros fatores, como palatabilidade da dieta (Grummer et al., 1990), motilidade do intestino e liberação de hormônios intestinais que controlam a saciedade (Allen, 2000; Araujo et al., 2010). Um período maior de adaptação às dietas com inclusão de AGPI, antes do confinamento, poderia reduzir esses efeitos negativos (Araujo et al., 2010) e potencializar a ação imunológica dos AGPI (Cooke et al., 2011).

O objetivo deste trabalho foi avaliar o efeito da adição de fontes de lipídeos no desempenho e nas respostas imunológicas de bovinos Nelore em confinamento.

\section{Material e Métodos}

O estudo foi realizado na Faculdade de Medicina Veterinária e Zootecnia, da Universidade Estadual Paulista, Campus de Botucatu, nas instalações da Fazenda Edgárdia, e o confinamento experimental foi conduzido no Departamento de Melhoramento e Nutrição Animal, conforme normas do Comitê de Ética (Protocolo n ${ }^{0} 34 / 2011$ CEUA).

Utilizou-se o delineamento experimental inteiramente casualizado, com medidas repetidas no tempo, composto pelas três dietas que caracterizaram os tratamentos: sem fonte adicional de lipídeo (CONTR); com fonte de lipídeos proveniente de coprodutos do algodão (GDESP); e com fonte de lipídeo protegido da degradação ruminal (GPROT), rico em ácidos graxos poli-insaturados Megalac E com sais de cálcio de ácido graxo de cadeia longa (Arm \& Hammer, Church \& Dwight Company, QGN, Rio de Janeiro, RJ, Brasil). O estudo foi dividido em duas fases: pré-confinamento e confinamento, tendo-se mantido os mesmos tratamentos nas duas fases.

Utilizaram-se120 animais machos da raça Nelore, não castrados, com peso vivo inicial (PVI) de $366,9 \pm 28,7 \mathrm{~kg}$, provenientes de recria em sistema de pastejo contínuo em pastagem de Urochloa brizantha 'Marandu' (Syn. Brachiaria brizantha 'Marandu'), com oferta total de $3,130 \mathrm{~kg}$. Na primeira fase, 30 dias antes do transporte para o confinamento, os animais - em jejum de 16 horas - foram pesados; entraram no experimento no dia 19 de julho de 2011 e foram distribuídos aleatoriamente nos três tratamentos (40 animais por piquete, com 12 ha por piquete e $1 \mathrm{~m}$ de cocho por animal). A ingestão foi limitada a 2,32, 2,15 e 2,00 kg por dia por animal, para os tratamentos CONTR, GDESP e GPROT, respectivamente. Os suplementos isoproteicos foram pronta e completamente consumidos pelos animais. Cada tratamento foi fornecido em um único piquete, com a intenção apenas de se verificar o ganho de peso, garantir o consumo dos suplementos e preparar os animais para posterior transporte para o confinamento.

Ao final desse período, os animais foram pesados sem estar em jejum, para que não se agravasse sua condição, em razão da somatória tempo de jejum com tempo de transporte; descontou-se $4 \%$ do peso vivo cheio, para obtenção do ganho de peso. Os animais foram carregados e transportados em caminhões tipo "truck" (20 por caminhão), por aproximadamente 16 horas, até o confinamento experimental do Departamento de Melhoramento e Nutrição Animal da FMVZ, Unesp, Botucatu, onde se iniciou a fase de confinamento em 19 de agosto de 2011.

Após o desembarque, os animais foram pesados novamente (em jejum proporcionado pelo transporte) e distribuídos de acordo com os tratamentos, cada um com 8 baias ( 5 animais por baia, com $10 \mathrm{~m}^{2}$ por animal e $1 \mathrm{~m}$ de cocho por animal), consideradas como unidades experimentais.

Formuladas pelo sistema Large Ruminant Nutrition System 1.0.12, nível 2, com protocolo de adaptação gradual, as dietas foram divididas em três, para um período de 14 dias: a primeira foi fornecida por 
4 dias, e as duas restantes por 5 dias cada uma, até atingir o nível de concentrado desejado para a dieta de terminação (Tabela 1). Os animais foram alimentados ad libitum duas vezes ao dia (às 8:00 h e às 15:00 h), com água constante, em bebedouros tipo australiano.

A ingestão de matéria seca (IMS) foi medida para cada baia, por meio da pesagem do alimento fornecido diariamente. A pesagem da sobra foi efetuada todos os dias, antes do trato da manhã, tendo-se feito posteriormente a média de ingestão por animal. Os dados de IMS também foram expressos em percentagem do peso vivo (PV).

Pesagens intermediárias a cada 21 dias, com jejum de sólidos de 16 horas, foram feitas para monitorar o ganho de peso diário (GPD) e ajustar a dieta, quando necessário. Durante o período experimental, a conversão alimentar (CA) foi calculada pela divisão da IMS total pelo ganho de PV total, e a eficiência alimentar (EA) foi calculada pela divisão do ganho de PV total pela IMS. O custo de ganho de peso foi calculado com base no custo para cada animal ganhar um quilo de PV durante o confinamento, tendo-se comparado os três tratamentos utilizados no experimento. $\mathrm{O}$ custo do ganho foi calculado conforme a fórmula: Custo ganho $=\left[\mathrm{IMS}(\mathrm{kg}) \times\right.$ custo $\mathrm{kg}^{-1}$ de MS da dieta $\left.(\mathrm{R} \$)\right] / \mathrm{GPD}(\mathrm{kg})$.

A partir de amostras de sangue colhidas por meio de punção da veia jugular dos animais, em tubos vacutainer que continham heparina sódica, determinaram-se os valores dos níveis plasmáticos de ceruloplasmina, haptoglobina, cortisol, fator de necrose tumoral alfa (TNF $\alpha)$, e interleucinas 1 e 6 (IL-1 e IL-6). As amostras foram coletadas nos dias: 0 , imediatamente antes do carregamento dos animais para transporte; 1, imediatamente após transporte e desembarque; 3, 7 e 14. Logo após a coleta, as amostras foram centrifugadas a $2.500 \mathrm{ppm}$, por $30 \mathrm{~min}$, para separação do plasma. O plasma foi removido para um tubo micrograduado e armazenado a $80^{\circ} \mathrm{C}$ no mesmo dia da coleta. Registrou-se também a temperatura retal dos animais, por meio de termômetro digital.

Para as concentrações de TNF $\alpha$, IL-1, IL-6 e cortisol, utilizaram-se kits comerciais de Elisa específicos para bovinos: para TNF $\alpha$, DuoSet Elisa (R\&D Systems, Inc., Minneapolis, MN, USA); para IL-1 e IL-6, Screening Set (Thermo Scientific, Rockford, IL, USA); e para

Tabela 1. Composição e conteúdo nutricional das dietas tratamento ${ }^{(1)}$ oferecidas aos animais durante a segunda fase (confinamento).

\begin{tabular}{|c|c|c|c|c|c|c|c|c|c|c|c|c|}
\hline \multirow[t]{2}{*}{ Ingredientes (\% MS) } & \multicolumn{3}{|c|}{ Adaptação I (4 dias e 55\%) } & \multicolumn{3}{|c|}{ Adaptação II (5 dias e $65 \%$ ) } & \multicolumn{3}{|c|}{ Adaptação III (5 dias e 75\%) } & \multicolumn{3}{|c|}{ Terminação (70 dias e $85 \%$ ) } \\
\hline & CONTR & GDESP & GPROT & CONTR & GDESP & GPROT & CONTR & GDESP & GPROT & CONTR & GDESP & GPROT \\
\hline Bagaço de cana cru & 15,20 & 15,20 & 15,13 & 15,94 & 15,10 & 15,84 & 15,20 & 15,00 & 15,63 & 15,50 & 14,85 & 15,43 \\
\hline Feno de Coast Cross & 29,32 & 29,34 & 29,39 & 18,84 & 19,40 & 19,33 & 10,13 & 9,60 & 9,79 & - & - & - \\
\hline Grão úmido de milho & 26,48 & 23,35 & 25,82 & 33,33 & 31,37 & 34,39 & 40,52 & 42,34 & 41,96 & 50,29 & 53,12 & 50,62 \\
\hline Polpa cítrica & 8,21 & 3,53 & 7,13 & 12,61 & 5,81 & 8,77 & 16,46 & 7,70 & 13,46 & 21,37 & 10,08 & 19,14 \\
\hline Farelo de amendoim & 18,00 & 10,56 & 17,83 & 16,20 & 8,48 & 16,74 & 14,56 & 6,05 & 14,17 & 9,43 & - & 9,51 \\
\hline Torta de algodão & - & 15,23 & - & - & 16,84 & - & - & 16,24 & - & - & 18,64 & - \\
\hline Megalac E & - & - & 1,84 & - & - & 1,93 & - & - & 1,83 & - & - & 1,85 \\
\hline Ureia & 0,46 & 0,49 & 0,52 & 0,58 & 0,52 & 0,53 & 0,63 & 0,71 & 0,72 & 0,95 & 0,98 & 0,99 \\
\hline Suplemento mineral $^{(2)}$ & 2,33 & 2,30 & 2,34 & 2,50 & 2,47 & 2,47 & 2,49 & 2,37 & 2,45 & 2,46 & 2,33 & 2,47 \\
\hline \multicolumn{13}{|l|}{ Conteúdo nutricional $^{(3)}$} \\
\hline Matéria seca (\%) & 76 & 76 & 76 & 74 & 75 & 74 & 73 & 73 & 73 & 71 & 71 & 71 \\
\hline Proteína bruta (\%) & 16,0 & 16,0 & 16,0 & 15,5 & 15,5 & 15,5 & 15,0 & 15,0 & 15,0 & 13,7 & 13,7 & 13,7 \\
\hline Extrato etéreo (\%) & 2,7 & 4,0 & 4,2 & 3,0 & 4,3 & 4,6 & 3,2 & 4,7 & 4,8 & 3,6 & 5,3 & 5,2 \\
\hline $\operatorname{FDN}(\%)^{(4)}$ & 38,3 & 42,6 & 37,4 & 30,8 & 35,9 & 30,5 & 24,2 & 28,1 & 23,6 & 17,0 & 21,4 & 16,5 \\
\hline FDNef $(\%)^{(5)}$ & 33 & 35 & 33 & 26 & 28 & 27 & 20 & 21 & 20 & 13 & 14 & 12 \\
\hline $\operatorname{NDT}(\%)^{(6)}$ & 69 & 68 & 70 & 72 & 71 & 75 & 75 & 75 & 77 & 80 & 80 & 82 \\
\hline $\mathrm{NEg},\left(\text { Mcal kg }{ }^{-1}\right)^{(7)}$ & 1,00 & 0,97 & 1,03 & 1,09 & 1,06 & 1,14 & 1,18 & 1,18 & 1,23 & 1,29 & 1,29 & 1,34 \\
\hline $\operatorname{CNF}(\%)^{(8)}$ & 40 & 34 & 39 & 47 & 41 & 46 & 54 & 49 & 53 & 63 & 57 & 62 \\
\hline Cálcio (\%) & 0,77 & 0,66 & 0,91 & 0,85 & 0,70 & 0,93 & 0,89 & 0,69 & 0,98 & 0,94 & 0,68 & 1,06 \\
\hline Fósforo (\%) & 0,34 & 0,44 & 0,33 & 0,34 & 0,46 & 0,34 & 0,34 & 0,45 & 0,33 & 0,32 & 0,46 & 0,32 \\
\hline
\end{tabular}

(1)CONTR, dieta controle; GDESP, dieta com gordura proveniente de torta de algodão; GPROT, dieta com adição de fonte de ácidos graxos poli-insaturados protegidos no rúmen (Megalac-E). ${ }^{(2)}$ Níveis de garantia: Ca, 16,0\%; P, 2,4\%; Na, 5,9\%; S, 2,8\%; Mg, 0,5\%; Zn, 1680 ppm; Mn, 1120 ppm; Cu, 560 ppm; I, 28 ppm; Se, 5,6 ppm; Co, 8,23 ppm; vitamina A, 73920 UI; e monensina, 1.000 ppm. ${ }^{(3)}$ Valores de acordo com formulação pelo LRNS $1.0 .12 .{ }^{\left({ }^{4}\right)}$ Fibra insolúvel em detergente neutro. ${ }^{(5)} \mathrm{FDN}$ fisicamente efetivo. ${ }^{(6)}$ Nutrientes digestíveis totais. ${ }^{(7)}$ Energia líquida de ganho. ${ }^{(8)}$ Carboidratos não fibrosos. 
cortisol, AccuBind (Monobind, Inc., Lake Forest, CA, USA). As ceruloplasminas foram determinadas de acordo com o proposto por Demetriou et al. (1974). A haptoglobina foi determinada por medição do complexo haptoglobina/hemoglobina, por estimativa das diferenças na atividade da peroxidase (Makimura \& Suzuki, 1982), e os resultados foram expressos em unidades arbitrárias a partir da leitura de absorção em $450 \mathrm{~nm} \times 100$. O coeficiente de variação intra e inter ensaio foram, respectivamente, 8,14 e $1,30 \%$, para ceruloplasmina, e 3,2 e 2,28\% para haptoglobina.

Os animais foram abatidos, em frigorífico comercial, após 84 dias de experimento, quando alcançaram o PV final (PVF) de $491 \pm 36,4 \mathrm{~kg}$, o que atendia às exigências do frigorífico. $\mathrm{O}$ rendimento de carcaça (RC) foi calculado a partir do peso da carcaça quente (PQC), dividido pelo PVF do animal. A percentagem de gordura visceral $(\mathrm{GV})$ na carcaça foi calculada pela quantidade (kg) de GV dividida pelo PCQ. A variável gordura visceral utilizada foi constituída pela gordura de mesentério somada à gordura renal e pélvica. Os pulmões foram separados da carcaça, para serem examinados por métodos de inspeção e palpação, pelos agentes do Serviço de Inspeção Federal (SIF). A avaliação foi realizada individualmente, e cada lóbulo foi inspecionado em busca de lesões compatíveis com quadros de broncopneumonia. Cada animal foi classificado pela presença ou não de lesão pulmonar, independentemente da severidade.

Para a avaliação da incidência de ruminite (IR), após o abate dos animais, os rúmens foram lavados. O epitélio ruminal foi classificado quanto à incidência de lesões (ruminites) e outras anormalidades, em escala de 0 a 10 pontos, conforme Bigham \& McManus (1975). Os abscessos hepáticos (AH) foram classificados de acordo com a severidade (Brink et al., 1990). Em ambos, IR e AH, a classificação foi feita pelos profissionais da Unesp Botucatu, treinados para este fim.

Para análise estatística, os dados foram inicialmente testados quanto à normalidade e heterogeneidade, antes de se proceder à análise de variância e, quando necessário, foram transformados. Para determinar os graus de liberdade do denominador, para os testes de efeitos fixos, os dados foram analisados com o procedimento Proc Mixed do SAS (SAS Institute, Cary, NC, EUA) e o método de aproximação de Satterthwaite.
O modelo estatístico para análises de desempenho, custo do ganho e características de carcaça contém os efeitos fixos do tratamento. Os dados foram analisados, usando-se a baia (tratamento) como efeito aleatório. A baia foi, ainda, considerada como unidade experimental, tendo-se avaliado o valor médio dos animais dentro dela. O PVI no pré-confinamento foi utilizado como covariável na análise de ganho de peso total e ganho de peso diário (GPD). O modelo estatístico usado para análise de cortisol, citocinas, proteínas de fase aguda e temperatura retal contém os efeitos fixos do tratamento, dias de coleta e interações resultantes. Os dados foram analisados, tendo-se a baia (tratamento) como efeito aleatório e dias como repetição. A estrutura de covariância utilizada foi autorregressiva, o que proporcionou o menor critério de informação de Akaike e, portanto, o melhor ajuste. Para a análise dos dados de IR, AH e broncopneumonias, utilizou-se o Proc Freq do SAS (SAS Institute, Cary, NC, EUA). Para a comparação entre as médias, utilizou-se o teste de Tukey, a 5\% de probabilidade.

\section{Resultados e Discussão}

Houve diferença entre os tratamentos quanto ao PVI, na fase de pré-condicionamento $(p=0,004)$, em razão da heterogeneidade dos lotes de animais (Tabela 2). Fatores que também contribuíram para esta diferença foram a estrutura da fazenda e a casualização do experimento. Observou-se maior PVF $(p<0,0001)$ no tratamento GDESP, relacionado ao maior GPD em comparação aos demais tratamentos $(p=0,002)$, além de diferenças nos PVI.

Durante a fase de confinamento, os animais do tratamento GDESP apresentaram maior PVI $(p<0,0001)$, seguido dos tratamentos CONTR e GPROT. Porém, aos 42 dias de confinamento, os tratamentos CONTR e GPROT apresentaram pesos similares, que permaneceram até ao $84^{\circ}$ dia. Os animais do tratamento GDESP foram os de maior peso, durante todo confinamento, tendo-se igualado ao GPROT somente aos 84 dias. A diferença de PVI está relacionada ao desempenho diferenciado durante a fase de pré-condicionamento, em que animais do tratamento GDESP apresentaram melhor GPD (Tabela 2).

Aos 21 dias de confinamento, observou-se GPD semelhante em todos os tratamentos $(\mathrm{p}=0,821)$. Ao final do experimento, os animais do tratamento CONTR 
apresentaram menor GPD do que os demais, e GPROT e GDESP permaneceram semelhantes quanto ao GPD. Os animais do GDESP apresentaram, do início ao fim do experimento, melhor IMS (em massa e, também, em percentagem de PV) do que os demais tratamentos,

Tabela 2. Desempenho de bovinos Nelore, durante fase de pré-confinamento e confinamento, alimentados com dieta suplementar de diferentes fontes de gordura ${ }^{(1)}$.

\begin{tabular}{|c|c|c|c|c|c|}
\hline \multirow[t]{2}{*}{ Característica } & \multicolumn{3}{|c|}{ Tratamento $^{(2)}$} & \multirow[t]{2}{*}{ EPM } & \multirow[t]{2}{*}{ Valor $p$} \\
\hline & CONTR & GDESP & GPROT & & \\
\hline & \multicolumn{5}{|c|}{ Fase de pré-confinamento } \\
\hline Peso vivo inicial (kg) & $366,66 a b$ & $369,09 \mathrm{a}$ & $365,02 b$ & 1,08 & 0,004 \\
\hline Peso vivo final (kg) & $370,91 b$ & $376,68 \mathrm{a}$ & $369,30 \mathrm{c}$ & 0,52 & $<0,0001$ \\
\hline Ganho de peso total $(\mathrm{kg})$ & $4,25 \mathrm{~b}$ & $7,58 \mathrm{a}$ & $4,28 \mathrm{~b}$ & 0,96 & 0,002 \\
\hline \multirow[t]{2}{*}{ Ganho de peso diário $(\mathrm{kg})$} & $0,14 \mathrm{~b}$ & $0,25 \mathrm{a}$ & $0,14 \mathrm{~b}$ & 0,03 & 0,002 \\
\hline & \multicolumn{5}{|c|}{ Fase de confinamento } \\
\hline Peso vivo inicial (kg) & $358,78 b$ & $362,40 \mathrm{a}$ & $352,07 \mathrm{c}$ & 1,00 & $<0,0001$ \\
\hline Perda de peso no $T^{(3)}(\mathrm{kg})$ & 12,13 & 15,82 & 16,83 & 1,40 & 0,081 \\
\hline Perda de peso no $\mathrm{T}^{(3)}(\%)$ & 3,27 & 4,20 & 4,56 & 0,37 & 0,081 \\
\hline \multicolumn{6}{|l|}{ Peso vivo final (kg) } \\
\hline $0-21$ & $403,55 \mathrm{a}$ & $406,83 a$ & $396,73 b$ & 1,40 & $<0,0001$ \\
\hline $0-42$ & $433,62 b$ & $449,61 \mathrm{a}$ & $435,23 b$ & 3,74 & 0,0002 \\
\hline $0-63$ & $465,87 b$ & $485,98 \mathrm{a}$ & $470,66 b$ & 4,75 & 0,0004 \\
\hline $0-84$ & $487,76 \mathrm{~b}$ & $506,37 \mathrm{a}$ & $493,81 \mathrm{ab}$ & 3,65 & 0,005 \\
\hline \multicolumn{6}{|l|}{ Ganho de peso diário $(\mathrm{kg})$} \\
\hline $0-21$ & 2,13 & 2,19 & 2,13 & 0,11 & 0,821 \\
\hline $0-42$ & $1,78 \mathrm{~b}$ & $2,11 \mathrm{a}$ & $1,98 \mathrm{ab}$ & 0,08 & 0,001 \\
\hline $0-63$ & $1,70 \mathrm{~b}$ & $1,98 \mathrm{a}$ & $1,88 \mathrm{ab}$ & 0,07 & 0,001 \\
\hline $0-84$ & $1,53 \mathrm{~b}$ & $1,73 \mathrm{a}$ & $1,69 \mathrm{a}$ & 0,04 & 0,008 \\
\hline \multicolumn{6}{|l|}{ IMS (kg) } \\
\hline $0-21$ & $8,40 \mathrm{~b}$ & $8,90 \mathrm{a}$ & $8,38 b$ & 0,16 & 0,004 \\
\hline $0-42$ & $9,29 b$ & $10,24 \mathrm{a}$ & $9,42 b$ & 0,23 & 0,0009 \\
\hline $0-63$ & $9,42 b$ & $10,63 a$ & $9,65 b$ & 0,25 & 0,0002 \\
\hline $0-84$ & $9,71 b$ & $10,70 \mathrm{a}$ & $9,83 b$ & 0,18 & 0,002 \\
\hline \multicolumn{6}{|l|}{ IMS (\%) do peso vivo } \\
\hline $0-21$ & $2,20 \mathrm{~b}$ & $2,32 \mathrm{a}$ & $2,24 \mathrm{ab}$ & 0,04 & 0,018 \\
\hline $0-42$ & $2,34 b$ & $2,52 \mathrm{a}$ & $2,39 b$ & 0,05 & 0,003 \\
\hline $0-63$ & $2,28 b$ & $2,51 \mathrm{a}$ & $2,34 b$ & 0,05 & 0,0004 \\
\hline $0-84$ & $2,29 \mathrm{~b}$ & $2,47 \mathrm{a}$ & $2,32 \mathrm{~b}$ & 0,03 & 0,004 \\
\hline \multicolumn{6}{|l|}{ Conversão alimentar } \\
\hline $0-21$ & 4,06 & 4,09 & 3,97 & 0,19 & 0,785 \\
\hline $0-42$ & $5,24 \mathrm{a}$ & $4,85 b$ & $4,80 \mathrm{~b}$ & 0,15 & 0,017 \\
\hline $0-63$ & 5,56 & 5,36 & 5,16 & 0,16 & 0,061 \\
\hline $0-84$ & $6,34 \mathrm{a}$ & $6,18 \mathrm{ab}$ & $5,84 \mathrm{~b}$ & 0,11 & 0,012 \\
\hline \multicolumn{6}{|l|}{ Eficiência alimentar } \\
\hline $0-21$ & 0,248 & 0,245 & 0,253 & 0,010 & 0,765 \\
\hline $0-42$ & $0,191 b$ & $0,206 a b$ & $0,209 a$ & 0,006 & 0,019 \\
\hline $0-63$ & 0,180 & 0,187 & 0,194 & 0,005 & 0,054 \\
\hline $0-84$ & $0,158 \mathrm{~b}$ & $0,162 \mathrm{ab}$ & $0,171 \mathrm{a}$ & 0,002 & 0,010 \\
\hline
\end{tabular}

${ }^{(1)}$ Médias com letras iguais, nas linhas, não diferem pelo teste de Tukey, a $5 \%$ de probabilidade. ${ }^{(2)}$ CONTR, dieta controle; GDESP, dieta com gordura proveniente de torta de algodão; GPROT, dieta com adição de fonte de ácidos graxos poli-insaturados protegidos no rúmen (Megalac-E).

${ }^{(3)} \mathrm{T}$, transporte. EPM, erro-padrão médio; IMS, ingestão de matéria seca. exceto aos 21 dias, quando a IMS (em percentagem de PV) foi semelhante à do tratamento GPROT. O tratamento CONTR não apresentou diferença em relação ao GPROT para as variáveis IMS (em massa, e em percentagem de PV).

Conforme Aferri et al. (2005), a inclusão de sais de cálcio de ácidos graxos, em dietas com elevada proporção de concentrado (81\%), para novilhos em confinamento, fez decrescer a ingestão de massa de matéria seca, em comparação à dieta com caroço de algodão, mas não apresentou efeito sobre o desempenho. Os resultados de Aferri et al. (2005) similares aos do presente estudo, em que, apesar da menor ingestão de massa de matéria seca pelos animais do grupo GPROT, em comparação ao GDESP, não se observou diferença no ganho de peso diário. Resultados semelhantes foram observados por Sutter et al. (2000), que constataram menor ingestão de massa de matéria seca de tourinhos Pardo Suíço, alimentados com gordura protegida (4,7\% da MS), em relação ao tratamento controle, sem alteração do ganho de peso. Outros autores também relataram diminuição da IMS e ganho de peso vivo, quando bovinos foram suplementados com fontes de gordura (Gibb et al., 2004; Pavan et al., 2007), particularmente, quando gorduras protegidas na forma de sabões de cálcio são utilizados (Simas et al., 1995; Bateman et al., 1996; Ngidi et al., 1990). Além disso, as fontes de ácidos graxos poli-insaturados (PUFA), na forma de sabões de cálcio ou não, são mais prejudiciais ao consumo do que fontes de ácidos graxos saturados (SFA) (Elliott et al., 1996; Allen, 2000).

A inclusão de lipídeos em dietas para ruminantes pode afetar a ingestão de matéria seca, que pode ser influenciada pelo tipo de lipídeos adicionados e pela quantidade e tipo de forragem oferecida (Allen, 2000). A suplementação de PUFA, como sabões de cálcio, pode prejudicar a IMS e afetar outros fatores, como a palatabilidade da dieta, a motilidade do intestino e liberação de hormônios intestinais que controlam a saciedade (Allen, 2000). Estudos mostram que a suplementação de lipídeos em até $6 \%$ (com base na MS), para ruminantes que recebem alto concentrado, não influencia negativamente a utilização de outros componentes da dieta (Kucuk et al., 2004; Atkinson et al., 2006; Hess et al, 2008).

No presente estudo, a estrutura do ingrediente lipídico adicionado para o grupo GPROT não deve ser considerado como fator limitante do consumo, já que 
o grupo CONTR também apresentou reduzida IMS, em comparação ao GDESP. O principal motivo para esta diferença da IMS deve estar associado à melhor palatabilidade da torta de algodão, em relação aos demais ingredientes das dietas.

Aos 84 dias de confinamento, a CA e EA foram melhores nos animais do tratamento GPROT em relação ao CONTR, porém, ambos os tratamentos não diferiram do tratamento GDESP. Na avaliação do uso de dietas com lipídeos, na terminação de bovinos em confinamento, Brandt Jr. \& Anderson (1995) encontraram aumento do GPD e melhor CA. Da mesma forma, Zinn \& Shen (1996) relataram que ocorreu melhor EA em bovinos e concluíram que esse fator se associa aos maiores teores de energia metabolizável nos lipídeos, em relação aos alimentos ricos em carboidratos e proteína. Os níveis de energia da dieta do tratamento GPROT foram maiores do que na dieta CONTR, o que pode ter proporcionado melhor EA para o tratamento GPROT. Ngidi et al. (1990) avaliaram a EA de dietas com inclusão de 2,4 ou $6 \%$ de gordura protegida e a EA de dietas sem gordura protegida, tendo observado tendência de melhoria desta característica com o uso da fonte lipídica.

Para as variáveis características de carcaça (peso de carcaça quente, gordura visceral, área de olho de lombo final, espessura de gordura subcutânea final) não se observou diferença $(\mathrm{p}>0,05)$. Isto corrobora os resultados de Fiorentini (2009), que avaliou efeito do grão de soja, gordura protegida (Megalac E) e óleo de soja sobre as características das carcaças e a qualidade da carne de novilhas terminadas em confinamento. Quanto ao custo por quilo de PV, o tratamento CONTR apresentou menor valor $(\mathrm{p}=0,042)$ do que o GPROT (R\$ 3,04 vs. R\$ 3,28), e o GDESP (R\$ 3,09) foi semelhante aos demais tratamentos. Este resultado ocorreu pelo fato de o custo do ingrediente gordura protegida ser maior, contudo devem-se levar em conta outros benefícios proporcionados pela dieta com lipídeos protegidos, como melhoria na CA e EA, além das variações anuais nos custos dos ingredientes, bem como custos no armazenamento de ingredientes com maior volume em relação a alimentos mais densos.

Observou-se interação $(p=0,01)$ entre tratamento e dias, quanto aos níveis de cortisol (Figura 1). No dia 0 (antes do embarque), o grupo GDESP apresentou menor valor de cortisol do que CONTR e GPROT, que não diferiram entre si. Não se constatou diferença entre os tratamentos nos dias 1 e 14, contudo, o GPROT apresentou níveis de cortisol mais elevados nos dias 3 e 7 . Os níveis de cortisol indicam que os animais apresentaram resposta ao desafio de estresse proporcionado pelo transporte, com valores acima dos níveis basais entre 5 e $10 \mathrm{ng} \mathrm{mL}^{-1}$ encontrados por alguns autores (Yoshida \& Nakao, 2005). No presente estudo, níveis de cortisol entre 28 e $51 \mathrm{ng} \mathrm{mL}^{-1}$ estão próximos das concentrações circulantes de cortisol de $50 \mathrm{ng} \mathrm{mL}^{-1}$, relatados em outros estudos com referência a bovinos estressados (Arthington et al., 2003; Cooke et al., 2010), e acima das concentrações de 13,9 ng $\mathrm{mL}^{-1}$ encontradas por Cooke \& Bohnert (2011), em estudo com bovinos com genética taurina.

Os valores de cortisol do dia 0 mostram o estresse decorrente do manejo que os animais sofreram antes do embarque; todos os tratamentos apresentaram valores mais altos do que aqueles considerados basais e que se mantiveram ao longo do experimento. Segundo Curley et al. (2008), o manejo dos animais estimula a secreção e concentrações circulantes de ACTH (hormônio adrenocorticotrófico) e cortisol. O tratamento CONTR e GPROT apresentaram valores de cortisol reduzidos, ao final do experimento, e no GDESP, os valores se mantiveram constantes.

Ocorreu interação $(p=0,01)$ entre tratamento e dias, quanto à temperatura retal (Figura 2), em que o tratamento GPROT apresentou menor valor do

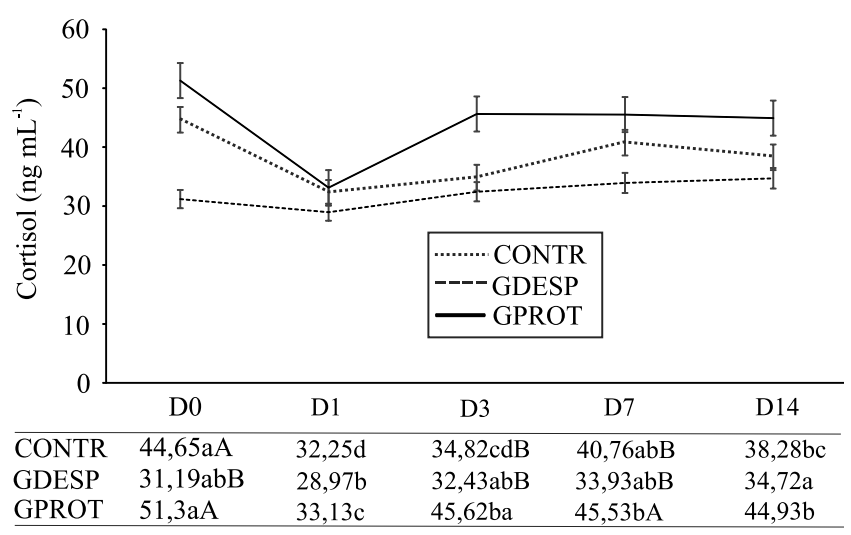

Figura 1. Interação entre tratamentos e dias para níveis de cortisol. Médias seguidas de letras iguais, minúsculas na linha e maiúsculas na coluna, não diferem pelo teste de Tukey, a 5\% de probabilidade. CONTR, dieta controle; GDESP, dieta com gordura proveniente de torta de algodão; GPROT, dieta com adição de fonte de ácidos graxos poliinsaturados protegidos no rúmen (Megalac-E). 
que o tratamento CONTR apenas no dia 0 de coleta $\left(39,3^{\circ} \mathrm{C}\right.$ vs. $\left.39,9^{\circ} \mathrm{C}\right)$, isto é, antes do embarque ao confinamento. Os menores valores de temperatura retal foram observados a partir do terceiro dia de confinamento, em todos os tratamentos, o que contraria os resultados encontrados por Cooke et al., (2011), que observaram os maiores valores de temperatura retal em novilhos, após três dias de chegada ao confinamento. $\mathrm{O}$ aumento da temperatura retal pode ser associado ao

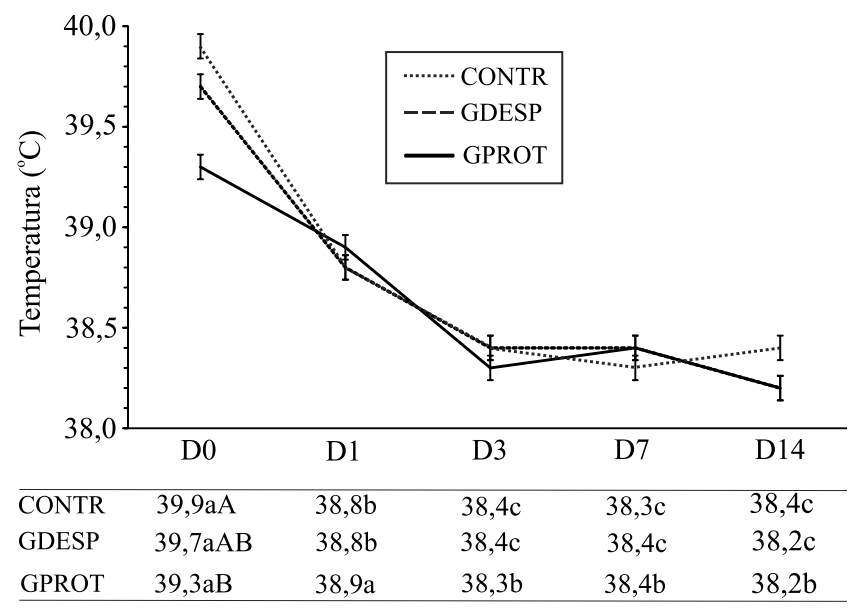

Figura 2. Interação entre tratamentos e dias para temperatura retal. Médias seguidas de letras iguais, minúsculas na linha e maiúsculas na coluna, não diferem pelo teste de Tukey, a 5\% de probabilidade. CONTR, dieta controle; GDESP, dieta com gordura proveniente de torta de algodão; GPROT, dieta com adição de fonte de ácidos graxos poli-insaturados protegidos no rúmen (Megalac-E). aumento dos níveis de TNF $\alpha$ (Cooke et al., 2012b), já que esta citocina é responsável pelo aumento da temperatura corporal e é importante estimulador da síntese hepática de proteínas de fase aguda (Yoshioka et al., 2002). Contudo, os níveis de TNF $\alpha$ permaneceram inalterados nos dias de coleta e não influenciaram a temperatura retal. Os maiores valores da temperatura retal, no dia do embarque dos animais ao confinamento, podem estar relacionados ao manejo aplicado antes do embarque, em razão da distância e do relevo acentuado entre o curral de manejo e os piquetes, onde os animais permaneceram durante 30 dias, o que influenciou também os níveis de cortisol.

Não se observou efeito $(\mathrm{p}>0,05)$ de dias de coleta, quanto aos níveis de TNF $\alpha$. Contudo, detectou-se efeito de tratamento $(p<0,0001)$, e os animais do tratamento CONTR apresentaram os maiores níveis de TNF $\alpha$ (Tabela 3). Diferenças entre GDESP e GPROT também foram detectadas, em que a concentração plasmática de $\mathrm{TNF} \alpha$ foi superior no tratamento GPROT. Os efeitos de tratamento detectados em concentrações plasmáticas de TNF $\alpha$ indicam que a suplementação de lipídeos na dieta, de modo geral, independentemente da fonte, pode aliviar a resposta inflamatória em bovinos de corte da raça Nelore. Rezamand et al. (2009) relataram que vacas lactantes, alimentadas com dietas suplementadas com farelo de camelina (rico em ácido linolênico), apresentaram expressão reduzida de IL-1 e TNF $\alpha$, em células mononucleares periféricas sanguíneas, em comparação a tratamentos não suplementados.

Não se detectou efeito de tratamento $(\mathrm{p}>0,05)$ quanto à IL-6. Ocorreu apenas o efeito de dias $(\mathrm{p}=0,0007)$, pois, no sétimo dia de confinamento, os animais

Tabela 3. Níveis de cortisol, citocinas, proteínas de fase aguda e temperatura retal de bovinos Nelore, de acordo com o tratamento e dias de coleta $^{(1)}$.

\begin{tabular}{|c|c|c|c|c|c|c|c|c|c|c|c|}
\hline \multirow[t]{2}{*}{ Itens } & \multicolumn{3}{|c|}{ Tratamento $^{(2)}$} & \multicolumn{5}{|c|}{ Dias de coleta } & \multirow[t]{2}{*}{ EPM } & \multicolumn{2}{|c|}{ Valor $\mathrm{p}$} \\
\hline & CONTR & GDESP & GPROT & 0 & 1 & 3 & 7 & 14 & & Tratamento & Dias \\
\hline $\operatorname{Cortisol}^{(3)}\left(\mathrm{ng} \mathrm{mL}^{-1}\right)$ & 38,2 & 32,2 & 44,1 & 42,4 & 31,5 & 37,6 & 40,1 & 39,3 & 3,78 & 0,049 & $<0,0001$ \\
\hline $\mathrm{TNF} \alpha^{(4)}\left(\mathrm{pg} \mathrm{mL} L^{-1}\right)$ & $5,11 \mathrm{a}$ & $5,01 \mathrm{c}$ & $5,04 \mathrm{~b}$ & 5,07 & 5,06 & 5,05 & 5,05 & 5,05 & 0,01 & $<0,0001$ & 0,238 \\
\hline Interleucina $6\left(\mathrm{pg} \mathrm{mL}^{-1}\right)$ & 6,68 & 6,59 & 6,55 & $6,60 \mathrm{bc}$ & $6,56 \mathrm{c}$ & $6,57 \mathrm{c}$ & $6,64 b$ & $6,70 \mathrm{a}$ & 0,06 & 0,287 & 0,0007 \\
\hline Interleucina $1 \beta^{(3)}\left(\mathrm{pg} \mathrm{mL}^{-1}\right)$ & 5,62 & 4,52 & 4,58 & 4,90 & 4,92 & 4,93 & 4,93 & 4,92 & 0,03 & $<0,0001$ & 0,276 \\
\hline Haptoglobina, $450 \mathrm{~nm} \times 100$ & $3,48 \mathrm{a}$ & $2,58 \mathrm{~b}$ & $2,34 \mathrm{~b}$ & 2,69 & 2,81 & 2,97 & 2,82 & 2,74 & 0,01 & 0,002 & 0,502 \\
\hline Ceruloplasmina $\left(\mathrm{mg} \mathrm{dL}^{-1}\right)$ & $15,75 \mathrm{a}$ & $12,97 \mathrm{~b}$ & $12,72 b$ & $11,08 \mathrm{~d}$ & $12,61 \mathrm{c}$ & $14,49 \mathrm{~b}$ & $15,02 b$ & $15,86 \mathrm{a}$ & 0,52 & 0,008 & $<0,0001$ \\
\hline Temperatura $\operatorname{retal} l^{(3)}\left({ }^{\circ} \mathrm{C}\right)$ & 38,8 & 38,7 & 38,6 & 39,6 & 38,8 & 38,3 & 38,4 & 38,3 & 0,06 & 0,398 & $<0,0001$ \\
\hline
\end{tabular}

${ }^{(1)}$ Médias com letras iguais, nas linhas, não diferem pelo teste de Tukey, a 5\% de probabilidade. (2)CONTR, dieta controle; GDESP, dieta com gordura proveniente de torta de algodão; GPROT, dieta com adição de fonte de ácidos graxos poli-insaturados protegidos no rúmen (Megalac-E). ${ }^{(3)}$ Interação entre tratamentos e dias, a 5\% de probabilidade. ${ }^{(4)}$ Fator de necrose tumoral alfa. EPM, erro-padrão médio. As interações se encontram nas Figuras 1,2 e 3. 
apresentaram aumento dos níveis plasmáticos de IL-6, com pico no décimo quarto dia (Tabela 3). Cooke \& Bohnert (2011) observaram pico de valores de IL-6 e IL- $1_{\beta}$ no terceiro dia de confinamento. O aumento das concentrações plasmáticas de IL-6 em relação às concentrações basais é atribuído aos efeitos catabólicos do cortisol nos tecidos corporais, e inclui a liberação de ácidos graxos não esterificados de tecidos adiposos, o que pode ser reconhecido pelo sistema imune como perturbação da homeostasia (Abbas et al., 2007). Níveis de cortisol, no presente experimento, apresentaram valores indicativos de estresse, o que sinaliza efeito substancial para promover o catabolismo nos tecidos e pode contribuir para o aumento nos níveis de IL-6.

Houve interação para a variável $\mathrm{IL}-1_{\beta}$ entre tratamento e dias $(\mathrm{p}<0,0001)$ (Figura 3$)$, em que os animais do GDESP e GPROT apresentaram valores inferiores de IL- $1_{\beta}$ do plasma sanguíneo, em relação aos do CONTR, e que permaneceram semelhantes, exceto no dia 7 de confinamento, quando os animais do tratamento GDESP apresentaram valores menores do que os do GPROT. Bovinos do grupo CONTR, de acordo com os dias de coleta, apresentaram início de

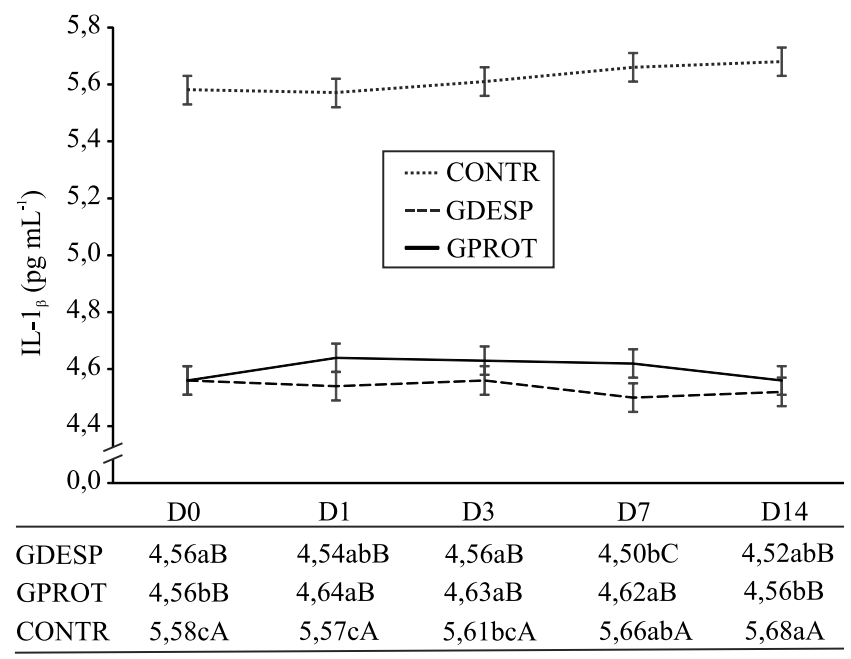

Figura 3. Interação entre tratamentos e dias para IL- $1_{\beta}$. Médias seguidas de letras iguais, minúsculas na linha e maiúsculas na coluna, não diferem pelo teste de Tukey, a $5 \%$ de probabilidade. CONTR, dieta controle; GDESP, dieta com gordura proveniente de torta de algodão; GPROT, dieta com adição de fonte de ácidos graxos poli-insaturados protegidos no rúmen (Megalac-E). aumento dos níveis de IL- $1_{\beta}$ a partir do terceiro dia, e o pico foi atingido no dia 14 . No tratamento GDESP, os níveis de IL- $1_{\beta}$ apresentaram tendência à pequena oscilação dos dados, no decorrer dos dias de coleta, com maior diferença entre os dias 3 e 7 . No tratamento GPROT, os animais apresentaram pequeno aumento no primeiro dia de confinamento, em relação à coleta pré-embarque. Estes níveis permaneceram semelhantes nos $3^{\circ}$ e $7^{\circ}$ dias, retornando a valores inferiores no $14^{\circ}$ dia.

É possível observar que a suplementação lipídica à dieta, independentemente da fonte, em todos os tratamentos, promoveu melhora dos parâmetros imunológicos de bovinos de corte da raça Nelore e reduziu os níveis de IL $1 \beta$, em relação à dieta controle sem inclusão de lipídeos (Rezamand et al., 2009; Cooke \& Bohnert, 2011) .

$\mathrm{O}$ efeito de tratamento $(\mathrm{p}=0,002)$ quanto à haptoglobina, com animais do CONTR, apresentou níveis mais elevados do que os demais tratamentos, que não diferiram entre si (Tabela 3 ). O mesmo efeito de tratamento $(p=0,008)$ foi constatado quanto aos níveis de ceruloplasmina, em que CONTR apresentou níveis mais elevados do que os demais tratamentos. Para esta variável, houve efeito de dias de coleta $(p<0,0001)$, tendo ocorrido aumento gradual dos níveis de ceruloplasmina até a coleta realizada no dia 14, enquanto os valores dos dias 3 e 7 foram semelhantes estatisticamente. As concentrações plasmáticas de ceruloplasmina e haptoglobina são normalmente correlacionadas e, apesar das diferenças entre tratamentos e entre dias de coleta, os valores encontrados se mostram muito próximos dos níveis normais (Cooke et al., 2009).

Os níveis de haptoglobina se encontram acima dos encontrados por Cooke et al. (2012a). Contudo, valores observados no tratamento CONTR foram próximos dos encontrados por Araujo et al. (2010), no tratamento sem inclusão de gordura, que apresentaram pico de haptoglobina no quarto dia de coleta para o mesmo tratamento. Gabay \& Kushner (1999) mostraram que os aumentos significativos de haptoglobina são detectados horas após estímulo inflamatório, ao passo que o pico das concentrações de haptoglobina é tipicamente observado entre 24 e 192 horas após a indução do estresse (Araujo et al., 2010; Cooke \& Bohnert, 2011). Contudo, não houve diferença $(p>0,05)$ dos níveis de haptoglobina nos dias de coleta, 
apesar de as concentrações de cortisol apresentarem valores altos, o que indica diferenças entre os dias de coleta, principalmente com o dia do embarque.

Cullens et al. (2005) não encontrou diferenças quanto aos valores de haptoglobina e ceruloplasmina em vacas em lactação, alimentadas com dietas suplementadas ou não com sais de cálcio de ácidos graxos. Cooke et al. (2011) encontraram efeitos de tratamento para estas variáveis e verificaram que os tratamentos que receberam lipídeos reduziram os valores das proteínas de fase aguda, quando os animais foram submetidos a estresse por transporte. Araujo et al. (2010) relataram que as concentrações de haptoglobina foram reduzidas em novilhas que receberam dietas à base de forragem enriquecida com Pufa, oferecida no pré-confinamento, em comparação com grupos controle, durante os primeiros oito dias após o transporte. Outros estudos, que utilizaram um modelo de transporte similar, para avaliar concentrações plasmáticas de proteínas de fase aguda até 28 dias após o transporte, relataram respostas ao tratamento a partir de três dias após o transporte (Arthington et al., 2005, 2008).

Não se observou diferença $(p=0,10)$ quanto à quantidade de incidência de ruminite entre os tratamentos (GDESP, 23; GPROT, 32; CONTR, 29). Dos 120 pulmões avaliados, 22,5\% $(\mathrm{n}=27)$ apresentaram lesão pulmonar, porém, não houve diferença significativa $(\mathrm{p}=0,15)$ entre os tratamentos. Quanto à severidade, apesar de os valores de incidência de ruminite permanecerem baixos, houve efeito $(p=0,001)$ entre os tratamentos, em que o GDESP apresentou menor valor (GDESP, 1,10; GPROT, 2,07; CONTR, 1,95). As principais causas de ruminite são processos fermentativos inadequados no interior do rúmen, que podem ser gerados tanto por quadros de acidose láctica ruminal aguda - em que o baixo $\mathrm{pH}$ associado à alta osmolaridade pode provocar lesões na parede ruminal, em especial nas papilas -, quanto por acidose branda, provocada por ácidos graxos de cadeia curta, cuja produção excessiva no interior do rúmen se acumula principalmente nas regiões ventrais do órgão, o que gera lesões corrosivas em sua parede (Owens et al., 1998). Apesar da lesão pulmonar, não houve diferença significativa $(p=0,15)$ entre os pulmões avaliados nos tratamentos. Em razão do baixo número de casos de abscessos hepáticos, não foi possível avaliar estatisticamente esta variável nos tratamentos.

\section{Conclusões}

1. O uso de lipídeos protegidos contra a degradação ruminal não influencia o ganho de peso no período de pré-confinamento (antes do transporte e entrada no confinamento), diferentemente do que se observa com o uso de torta de algodão, que proporciona maior ganho de peso.

2. Animais alimentados com dieta suplementar de lipídeos protegidos, durante o período de confinamento, apresentam desempenho semelhante aos alimentados com dieta suplementar de torta de algodão, além de apresentar menor ingestão de massa de matéria seca.

3. As fontes de lipídeos não influenciam as características de carcaça.

4. A adição de lipídeos à dieta de animais confinados, independentemente da fonte (naturais ou inertes no rúmen), pode promover melhoria em parâmetros imunológicos do animal e reduzir os níveis de citocinas pro-inflamatórias, como TNF $\alpha$ e IL- $1_{\beta}$, e proteínas de fase aguda, como ceruloplasmina e haptoglobina, em relação à dieta sem inclusão de lipídeos.

5. O uso de torta de algodão na alimentação de bovinos confinados influencia positivamente as condições de saúde ruminal, ao diminuir o índice de lesões no órgão.

\section{Agradecimentos}

À Fundação de Amparo à Pesquisa do Estado de São Paulo (Fapesp), pelo apoio financeiro (processo 2012/00884-3) e pela bolsa concedida (processo 2011/01715-8).

\section{Referências}

ABBAS, A.K.; LICHTMAN, A.H.; PILLAI, S. Cellular and molecular immunology. $6^{\text {th }}$ ed. Philadelphia: Saunders, 2007. 572p.

AFERRI, G.; LEME, P.R.; SILVA, S. da L. e; PUTRINO, S.M.; PEREIRA, A.S.C. Desempenho e características de carcaça de novilhos alimentados com dietas contendo diferentes fontes de lipídios. Revista Brasileira de Zootecnia, v.34, p.1651-1658, 2005. DOI: $10.1590 / \mathrm{S} 1516-35982005000500027$.

ALLEN, M.S. Effects of diet on short term regulation of feed intake by lactating dairy cattle. Journal of Dairy Science, v.83, p.1598-1624, 2000. DOI: 10.3168/jds.S0022-0302(00)75030-2.

ARAUJO, D.B.; COOKE, R.F.; HANSEN, G.R.; STAPLES, C.R.; ARTHINGTON, J.D. Effects of rumen protected polyunsaturated fatty acid supplementation on performance and physiological responses of growing cattle after transportation and feedlot entry. 
Journal of Animal Science, v.87, p.4120-4132, 2010. DOI: 10.2527/jas.2009-2684.

ARTHINGTON, J.D.; EICHERT, S.D.; KUNKLE, W.E.; MARTINS, F.G. Effect of transportation and commingling on the acute phase protein response, growth, and feed intake of newly weaned beef calves. Journal of Animal Science, v.81, p.11201125,2003

ARTHINGTON, J.D.; QIU, X.; COOKE, R.F.; VENDRAMINI, J.M.B.; ARAUJO, D.B.; CHASE JR., C.C.; COLEMAN, S.W. Effects of preshipping management on measures of stress and performance of beef steers during feedlot receiving. Journal of Animal Science, v.86, p.2016-2023, 2008. DOI: 10.2527/jas.2008-0968.

ARTHINGTON, J.D.; SPEARS, J.W.; MILLER, D.C. The effect of early weaning on feedlot performance and measures of stress in beef calves. Journal of Animal Science, v.83, p.933-939, 2005.

ATKINSON, R.L.; SCHOLLJEGERDES, E.J.; LAKE, S.L.; NAYIGIHUGU, V.; HESS, B.W.; RULE, D.C. Site and extent of digestion, duodenal flow, and intestinal disappearance of total and esterified fatty acids in sheep fed a high concentrate diet supplemented with high linoleate safflower oil. Journal of Animal Science, v.84, p.387-396, 2006.

BATEMAN, H.G.; SPAIN, J.N.; ELLERSIECK, M.R. Influence of by-product feeds and tallow on lactation performance of Holstein cows during two seasons. Journal of Dairy Science, v.79, p.114120, 1996. DOI: 10.3168/jds.S0022-0302(96)76341-5.

BIGHAM, M.L.; MCMANUS, W.R. Whole wheat grain feeding of lambs. V. Effects of roughage and wheat grain mixtures. Australian Journal of Agricultural Research, v.26, p.1053-1062, 1975. DOI: 10.1071/AR9751053.

BRANDT JR., R.T.; ANDERSON, S.J. Use of supplemental fat to optimize net energy intake by feedlot cattle. In: SYMPOSIUM: INTAKE BY FEEDLOT CATTLE, 1995, Stillwater. Proceedings. Stillwater: Oklahoma State University, 1995. p.303-311.

BRINK, D.R.; LOWRY, S.R.; STOCK, R.A.; PARROTT, J.C. Severity of liver abscesses and efficiency of feed utilization of feedlot cattle. Journal of Animal Science, v.68, p.1201-1207, 1990 .

COOKE, R.F.; ARTHINGTON, J.D.; AUSTIN, B.R.; YELICH, J.V. Effects of acclimation to handling on performance, reproductive, and physiological responses of Brahman-crossbred heifers. Journal of Animal Science, v.87, p.3403-3412, 2009. DOI: $10.2527 /$ jas.2009-1910.

COOKE, R.F.; BOHNERT, D.W. Bovine acute-phase response after corticotrophin-release hormone challenge. Journal of Animal Science, v.89, p.252-257, 2011. DOI: 10.2527/jas.2010-3131.

COOKE, R.F.; BOHNERT, D.W.; MORIEL, P.; HESS, B.W.; MILLS, R.R. Effects of polyunsaturated fatty acid supplementation on forage digestibility, performance, and physiological responses of feeder cattle. Journal of Animal Science, v.89, p.3677-3689, 2011. DOI: $10.2527 /$ jas.2010-3515.

COOKE, R.F.; BOHNERT, D.W.; CAPPELLOZZA, B.I.; MUELLER, C.J.; DELCURTO, T. Effects of temperament and acclimation to handling on reproductive performance of Bos taurus beef females. Journal of Animal Science, v.90, p.3547-3555, 2012b. DOI: $10.2527 /$ jas.2011-4768.
COOKE, R.F.; CARROLL, J.A.; DAILEY, J.; CAPPELLOZZA, B.I.; BOHNERT, D.W. Bovine acute-phase response after different doses of corticotropin-releasing hormone challenge. Journal of Animal Science, v.90, p.2337-2344, 2012a. DOI: 10.2527/ jas.2011-4608.

COOKE, R.F.; SCARPA, A.; NERY, F.; COOKE, F.; BOHNERT, D.W. Effects of polyunsaturated fatty acid (PUFA) supplementation on forage intake and digestibility in beef cows. In: BEEF research report. Corvallis: Oregon State University, 2010. Available at: $<$ http://beefcattle.ans.oregonstate.edu $>$. Accessed on: 20 Sept. 2010.

CULLENS, F.C.; STAPLES, C.R.; BILBY, T.R.; SILVESTRE, F.; BARTOLOME, J.; SOZZI, A.; BADINGA, L.; THATCHER, W.W.; ARTHINGTON, J.D. Effect of timing of initiation of fat supplementation on milk production, plasma hormones and metabolites, and conception rates of Holstein cows in summer. Journal of Animal Science, v.82, p.308, 2005.

CURLEY JR., K.O.; NEUENDORFF, D.A.; LEWIS, A.W.; CLEERE, J.J.; WELSH JR, T.H.; RANDEL, R.D. Functional characteristics of the bovine hypothalamic-pituitary-adrenal axis vary with temperament. Hormones and Behavior, v.53, p.20-27, 2008. DOI: 10.1016/j.yhbeh.2007.08.005.

DEMETRIOU, J.A.; DREWES, P.A.; GIN, J.B. Ceruloplasmin. In: HENRY, R.J.; CANNON, D.C.; WINKELMAN, J.W. (Ed.). Clinical chemistry: principles and techniques. $2^{\text {nd }}$ ed. Hagerstown: Harper and Row, 1974. p.857-864.

ELLIOTT, J.P.; DRACKLEY, J.K.; WEIGEL, D.J. Digestibility and effects of hydrogenated palm fatty acid distillate in lactating dairy cows. Journal of Dairy Science, v.79, p.1031-1039, 1996. DOI: 10.3168/jds.S0022-0302(96)76456-1.

FARRAN, T.B.; REINHARDT, C.D.; BLASI, D.A.; MINTON, J.E.; ELSASSER, T.H.; HIGGINS, J.J.; DROUILLARD, J.S. Source of dietary lipid may modify the immune response in stressed feeder cattle. Journal of Animal Science, v.86, p.13821394, 2008. DOI: 10.2527/jas.2007-0116.

FIORENTINI, G. Fontes lipídicas na terminação de novilhas. 2009.57p. Dissertação (Mestrado) - Faculdade de Ciências Agrárias e Veterinárias, Universidade Estadual Paulista, Jaboticabal.

GABAY, C.; KUSHNER, I. Acute-phase proteins and other systemic responses to inflammation. The New England Journal of Medicine, v.340, p.448-454, 1999. DOI: 10.1056/ NEJM199902113400607.

GIBB, D.J.; OWENS, F.N.; MIR, P.S.; MIR, Z.; IVAN, M.; MCALLISTER, T.A. Value of sunflower seed in finishing diets of feedlot cattle. Journal of Animal Science, v.82, p.2679-2692, 2004.

GRUMMER, R.R.; HATFIELD, M.L.; DENTINE, M.R. Acceptability of fat supplements in four dairy herds. Journal of Dairy Science, v.73, p.852-857, 1990. DOI: 10.3168/JDS.S00220302(90)78739-5.

HESS, B.W.; MOSS, G.E.; RULE, D.C. A decade of developments in the area of fat supplementation research with beef cattle and sheep. Journal of Animal Science, v.86, p.188-204, 2008. 
KUCUK, O.; HESS, B.W.; RULE, D.C. Soybean oil supplementation of a high concentrate diet does not affect site and extent of organic matter, starch, neutral detergent fiber, or nitrogen digestion, but influences both ruminal metabolism and intestinal flow of fatty acids in limit fed lambs. Journal of Animal Science, v.82, p.29852994, 2004.

LOERCH, S.C.; FLUHARTY, F.L. Physiological changes and digestive capabilities of newly received feedlot cattle. Journal of Animal Science, v.77, p.1113-1119, 1999.

MAKIMURA, S.; SUZUKI, N. Quantitative determination of bovine serum haptoglobin and its elevation in some inflammatory diseases. The Japanese Journal of Veterinary Science, v.44, p.15-21, 1982. DOI: 10.1292/jvms 1939.44.15.

NGIDI, M.E.; LOERCH, S.C.; FLUHARTY, F.L.; PALMQUIST, D.L. Effects of calcium soaps of long chain fatty acids on feedlot performance, carcass characteristics and ruminal metabolism of steers. Journal of Animal Science, v.68, p.2555-2565, 1990.

OWENS, F.N.; SECRIST, D.S.; HILL, W.J.; GILL, D.R. Acidosis in cattle: a review. Journal of Animal Science, v.76, p.275-286, 1998.

PAVAN, E.; DUCKETT, S.K.; ANDRAE, J.G. Corn oil supplementation to steers grazing endophyte-free tall fescue. I. Effects on in vivo digestibility, performance, and carcass traits. Journal of Animal Science, v.85, p.1330-1339, 2007. DOI: 10.2527/jas.2006-623.

RADOSTITS, O.M.; GAY, C.C.; HINCHCLIFF, K.W.; CONSTABLE, P.D. Veterinary Medicine. $10^{\text {th }}$ ed. Philadelphia: Saunders, 2007. p.935-946.
REZAMAND, P.; HUNT, K.M.; WILLIAMS, J.E.; SHIELDS, S.; MCGUIRE, M.A. Effects of trans fatty acids on markers of inflammation. The Faseb Journal, v.23, p.910-916, 2009.

SIMAS, J.M.; HUBER, J.T.; WU, Z.; CHEN, K.H.; CHAN, S.C.; THEURER, C.B.; SWINGLE, R.S. Influence of steam-flaked sorghum grain and supplemental fat on performance of dairy cows in early lactation. Journal of Dairy Science, v.78, p.1526-1533, 1995. DOI: 10.3168/jds.S0022-0302(95)76774-1.

SUTTER, F.; CASUTT, M.M.; OSSOWSKI, D.A.; SCHEEDER, M.R.L.; KREUZER, M. Comparative evaluation of rumenprotected fat, coconut oil and various oilseeds supplemented to fattening bulls. 1. Effects on growth, carcass and meat quality. Archives of Animal Nutrition, v.53, p.1-23, 2000. DOI: 10.1080/17450390009381935.

YOSHIDA, C.; NAKAO, T. Response of plasma cortisol and progesterone after ACTH challenge in ovariectomized lactating dairy cows. Journal of Reproduction and Development, v.51, p.99-107, 2005. DOI: 10.1262/jrd.51.99.

YOSHIOKA, M.; WATANABE, A.; SHIMADA, N.; MURATA, H.; YOKOMIZO, Y.; NAKAJIMA, Y. Regulation of haptoglobin secretion by recombinant bovine cytokines in primary cultured bovine hepatocytes. Domestic Animal Endocrinology, v.23, p.425-433, 2002. DOI: 10.1016/S0739-7240(02)00174-1.

ZINN, R.A.; SHEN, Y. Interaction of dietary calcium and supplemental fat on digestive function and growth performance in feedlot steers. Journal of Animal Science, v.74, p.2303-2309, 1996. 